\title{
Measurement of ultralow losses in an optical interferometer
}

\author{
G. Rempe, R. J. Thompson, and H. J. Kimble
}

Norman Bridge Laboratory of Physics, M.S. 12-33, California Institute of Technology, Pasadena, California 91125

\author{
R. Lalezari
}

PMS Electro-Optics, 1855 South 57th Court, Boulder, Colorado 80301

Received October 23, 1991

\begin{abstract}
Characterizations of low-loss mirrors by measurements of cavity-decay time and of cavity finesse are reported near $850 \mathrm{~nm}$. The lowest observed mirror loss is $1.6 \times 10^{-6}$ (transmission plus absorption and scatter), which corresponds to a reflectivity of 0.9999984 and to a cavity finesse of $1.9 \times 10^{6}$.
\end{abstract}

High-reflectivity mirrors with small scatter and absorption losses are important for a wide range of applications in optical science. In recent years there has been considerable progress both in the development of high-quality superpolished substrates with surface roughness at the angstrom level and in the growth of extremely low-loss thin-film dielectric coatings. Indeed, interferometers with total mirror loss $L$ (transmission plus scatter and absorption) below $8 \times 10^{-5}$ are commercially available, and cavities with losses of the order of $3 \times 10^{-5}$ are routinely employed in a research environment. Beyond this current state of the art, we report in this Letter a significant step toward mirrors with yet even lower losses. In particular, from observations of cavitydecay rate and of cavity finesse for a sphericalmirror interferometer formed by superpolished mirrors with specially fabricated dielectric coatings, we find that the loss for an individual mirror is $L \simeq 1.6 \times 10^{-6}$ and hence that the reflectivity is $R=1-L \simeq 0.9999984$ near $850 \mathrm{~nm}$. Furthermore reflectivities higher than 0.999996 are inferred over the wavelength range from 830 to $880 \mathrm{~nm}$. Measurements of the cavity reflection properties suggest that the partitioning of loss between mirror transmission $T$ and scatter and absorption loss $A$ is such that $T=0.5 \times 10^{-6}$ and $A=1.1 \times 10^{-6}$ for each mirror at $850 \mathrm{~nm}$.

For our investigation, two different sets of mirror substrates were employed. The first set was manufactured by General Optics with the substrates fabricated from fused silica with one side flat and the other side superpolished to a concave surface of radius of curvature $173 \mathrm{~mm}$. The central $2.5 \mathrm{~mm}$ of each substrate was polished to provide a surface sphericity of much better than $\lambda / 10$ and a surface microroughness less than $0.1 \mathrm{~nm}{ }^{1}$ The second set of substrates was fabricated to similar specifications at PMS Electro-Optics from BK-7 glass into a plano-concave form with a radius of curvature of $1 \mathrm{~m}$.

All mirror coatings for our research were produced by PMS Electro-Optics. An automated reactive ion-beam sputtering process was used to deposit a 41 quarter-wave $\mathrm{Ta}_{2} \mathrm{O}_{5} / \mathrm{SiO}_{2}$ coating. ${ }^{2}$ The films were sputtered from high-purity oxide targets in a cryogenically pumped vacuum chamber that was backfilled with partially ionized oxygen to achieve stoichiometric films. Cleanliness and lack of point defects were critical in achieving the targeted loss levels $\left(\sim 1 \times 10^{-6}\right)$. All substrate cleaning procedures and deposition parameters had been optimized at PMS by using cavity decay measurements (with an apparatus similar to the one described in this Letter) and by employing a surface analysis system. ${ }^{3}$

As illustrated in Fig. 1, the instrumentation for our measurements of cavity-decay time is relatively simple. ${ }^{4}$ Mirrors M1 and M2 are mounted in a clean environment into a sealed Teflon holder with a separation $d=(4.0 \pm 0.2) \mathrm{mm}$ between the mirrors. Small changes in the length of the resulting (symmetric) spherical mirror cavity are made by pushing on one of the substrates with a piezoelectric transducer PZT to compress slightly the Teflon spacer. The cavity is excited with light from a cw Ti:sapphire ( $\mathrm{Ti}: \mathrm{Al}_{2} \mathrm{O}_{3}$ ) laser that is mode matched with lens $\mathrm{L} 1$ to the $\mathrm{TEM}_{00}$ transverse mode of the resonator. Light transmitted by the cavity is imaged by lens L 2 onto a circular aperture A with a magnification of $5 \times$ and is detected by the photomultiplier tube $\mathrm{D}$.

To record the decay time of the intracavity intensity (the ring-down measurement), the length of the cavity is slowly scanned with the piezoelectric transducer until the resonance frequency of a TEM $_{00}$ longitudinal mode of the cavity begins to come into resonance with the incident laser. At a preset threshold, the detection of transmitted light by detector D triggers the acousto-optic switch (AOS), which after a delay of $\sim 300 \mathrm{~ns}$ turns off the incident laser in approximately $45 \mathrm{~ns}$. The subsequent decay of the cavity output is recorded on a digital storage oscilloscope. The time response of the system is characterized operationally by using a relatively low-finesse cavity in place of the cavity with supermirrors and by directly examining the light after the AOS with no optical cavity.

A typical measurement of the cavity ring down 


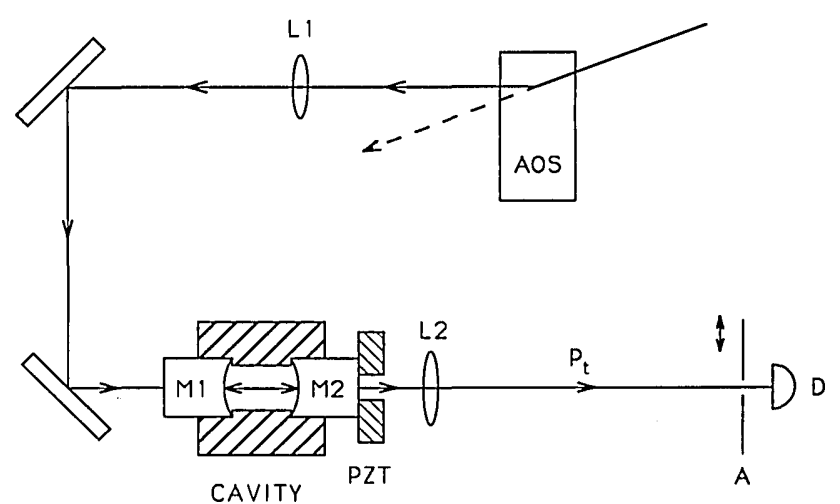

Fig. 1. Diagram of the apparatus used for measurement of cavity-decay time and finesse.

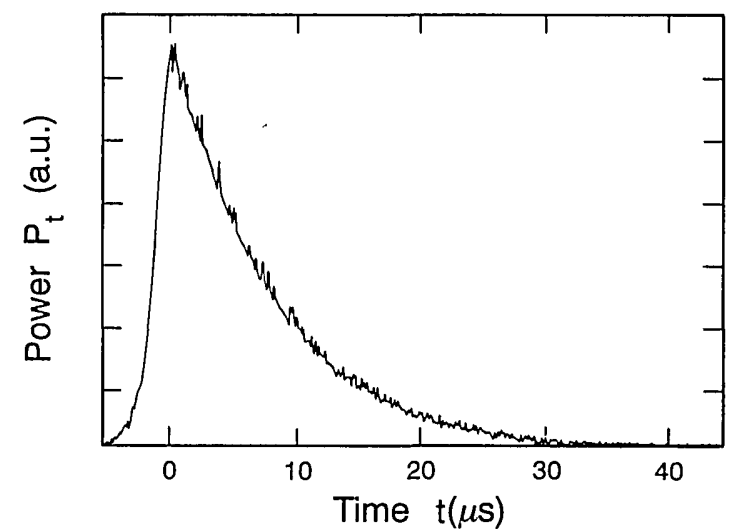

Fig. 2. Transmitted power $P_{t}$ monitored with detector D versus time $t$. The initial rise in $P_{t}$ for $t<0$ corresponds to the filling of the cavity as the cavity length scans into resonance. At $t=0, P_{t}$ crosses a predetermined threshold and the input laser is switched off by the AOS in Fig. 1. The subsequent decay of the intracavity field is then exhibited for $t>0$. This curve is an average of 100 single decays for an excitation wavelength of $852 \mathrm{~nm}$.

after the incident laser has been switched off is shown in Fig. 2, which is taken for two General Optics substrates. As expected for a cavity for which the round-trip time $\Delta t \equiv 2 d / c$ is much smaller than the decay time $\tau$, the decay of the transmitted power is approximately exponential, with the time taken for the transmitted power to decay to $1 / e$ of its initial value given by

$$
\tau=\frac{1}{2} \frac{\Delta t}{L}
$$

where the total loss per mirror is $L=1-R$, with $R$ the reflectivity of the mirrors (which are assumed to be identical). Note that $L$ is the sum of all losses at each mirror and includes the (useful) transmission loss $T$ as well as losses $A$ as a result of scatter and absorption (i.e., $L=T+A$ ). For the particular measurement of Fig. 2, the decay time $\tau=8.2 \mu \mathrm{s}$, so that the inferred mirror loss is $L=1.6 \times 10^{-6}$. Stated somewhat differently, since the quality factor $Q$ for a resonator is defined as $2 \pi$ times the ratio of stored energy to energy dissipation per cycle, we have $Q=\omega \tau$, with the optical frequency $\omega=2 \pi c / \lambda$ and $\tau$ as the energy decay time as in Eq. (1). We then have $Q \simeq 1.8 \times 10^{10}$ for our optical cavity of length $d=4 \mathrm{~mm}$. Data such as in Fig. 2 for the $\mathrm{TEM}_{00}$ mode are largely independent of the diameter of the aperture A over a range both smaller than and larger than the waist in the image plane.

To characterize further the mirror performance, we present in Fig. 3 the results of measurements made as in Fig. 2 with wavelengths over the range $790 \mathrm{~nm} \leq \lambda \leq 880 \mathrm{~nm}$, with the tuning range determined by that of our laser. These data show that the coatings have good performance over a reasonably broad bandwidth with reflectivities higher than 0.999996 inferred over the range $830 \mathrm{~nm} \leq$ $\lambda \leq 880 \mathrm{~nm}$. With the exception of the point at $837 \mathrm{~nm}$, all these data are for two General Optics substrates with the setup of Fig. 1. The point at $837 \mathrm{~nm}$ is obtained in a separate experiment with a semiconductor diode laser as the light source and a cavity of length $d^{\prime}=100 \mathrm{~mm}$ with PMS mirrors of 1-m radii. This measurement agrees relatively well with the trend of the other data. The explanation for the asymmetry evident in the figure is uncertain, especially since it is considerably larger than that expected from the calculated variation in transmissivity for the dielectric stack. For measured transmission values $T$ for $\lambda \leq 750 \mathrm{~nm}$ and $\lambda \geq 935 \mathrm{~nm}$ we infer that $T$ should increase from a minimum of $1.4 \times 10^{-6}$ at $833 \mathrm{~nm}$ to a value of $2 \times 10^{-6}$ at 812 and $855 \mathrm{~nm}$.) The asymmetry is also much larger than one might deduce from a $\lambda^{-4}$ dependence of Rayleigh scattering. Presumably there is some yet-to-be-identified wavelengthdependent absorption in the coating materials. Clearly a more detailed investigation of the nature of the loss mechanisms is warranted.

While our discussion thus far has centered on measurements of the decay of the $\mathrm{TEM}_{00}$ mode of the cavity, we have also investigated decay rates for the $\mathrm{TEM}_{01}$ mode, which could be distinguished operationally from other modes by imaging one of its lobes onto the aperture of Fig. 1 . While there is considerable variability in these measurements, the losses are typically $40 \%$ higher for this higher-order transverse mode. This increase in loss may simply

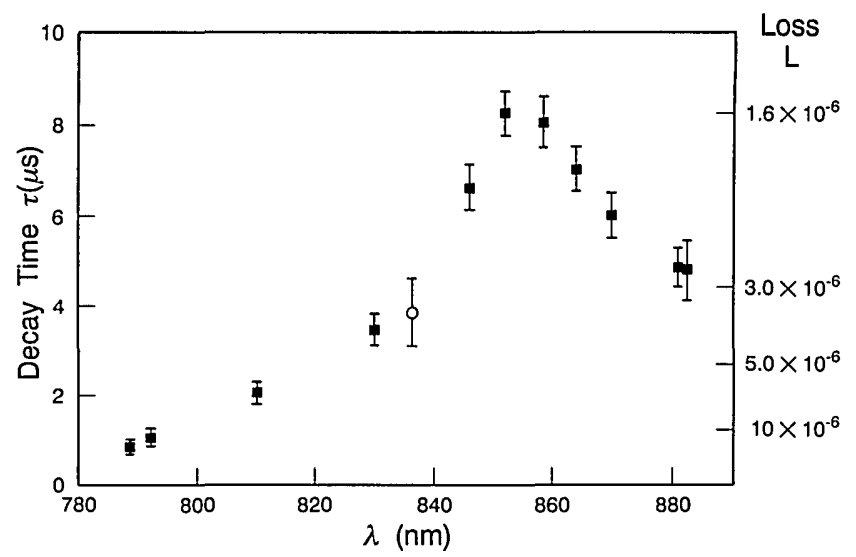

Fig. 3. Decay time $\tau$ for intracavity power versus excitation wavelength $\lambda$. The right-hand scale gives the inferred loss $L=d / c \tau$, with $d=4 \mathrm{~mm}$ as the cavity length. The point at $\lambda=837 \mathrm{~nm}$ was taken at PMS with a diode laser as discussed in the text and is referenced only to the scale for $L$. 
reflect some unimportant anomaly in the inhomogeneous distribution of defects on the mirror substrate or coating or it may be fundamentally related to the nature of the spatial distribution of the losses. An investigation of these issues is continuing with more systematic measurements of cavity decay times taken at different positions over the aperture of the mirrors for various spot sizes and transverse modes.

Beyond a characterization of the cavity properties in terms of the loss $L$ determined by way of decay time, measurements such as those in Figs. 2 and 3 unfortunately shed little light on the question of the partitioning of losses among the possibilities of transmission, scatter, and absorption. Indeed, it is important to note that the requirements for obtaining high finesse are somewhat more stringent than those for obtaining a long ring-down time, since a high finesse dictates that the spatial coherence of the intracavity field be maintained after repeated reflections. On the other hand, a long cavity-decay time requires only that energy be confined within the cavity in a fashion that is less dependent on small aberrations of the wave front. In particular, it is possible to conceive of small-angle scattering events that degrade the finesse but which leave light confined within the cavity and hence able to contribute to a long ring-down time. We have therefore made additional measurements to determine the cavity finesse of the interferometer. Because the linewidth of the cavity with $d=4 \mathrm{~mm}$ is so narrow (inferred to be approximately $20 \mathrm{kHz}$ ), we have introduced a much shorter cavity of length $d^{\prime}=$ $130 \mu \mathrm{m}$ into the setup shown in Fig. 1 (an accurate determination of the cavity length is made from observations of the transverse-mode spacing of the cavity). While the ring-down measurements described above are basically insensitive to fluctuations of cavity length, this is of course not the case for finesse measurements, so that some effort was made to isolate the shorter cavity from acoustic noise of the optical table and to damp mirror vibrations in the frequency range above $1 \mathrm{kHz}$. With the length of the isolated cavity held approximately constant, the frequency of the incident laser is swept with a fast linear ramp, with frequency calibration provided relative to the saturated resonances in an auxiliary cell of cesium vapor. A knowledge of the linewidth $\delta \nu$ of the cavity then yields the finesse $F=\left(c / 2 d^{\prime} \delta \nu\right)$, which for excitation near $852 \mathrm{~nm}$ gives $F=(1.9 \pm 0.1) \times 10^{6}$ as determined from $\delta \nu=600 \mathrm{kHz}$. This result is in good agreement with the value of finesse inferred from the cavity ring-down measurement, namely, $F=\pi / L \simeq 2 \times$ $10^{6}$, which indicates that a single number $L$ suffices to specify both $F$ and $\tau$.

As for the issue of the partitioning of the mirror losses between $T$ and $A$ (transmission and absorption plus scatter, respectively), we have recorded the reflection dip from the cavity, with $R_{0} \equiv$ (reflected power on resonance)/(reflected power off resonance). For excitation matched to the $\mathrm{TEM}_{00}$ transverse mode we find that $R_{0}=0.45 \pm 0.04$, from which we infer that $T / A \simeq 0: 45 \pm 0.1$, assuming identical mirrors. Given that $(A+T) \simeq 1.6 \times 10^{-6}$ as determined either from measurements of cavity decay or from scan finesse, we thus find that $T \simeq 0.5 \times 10^{-6}\left[0.5\right.$ parts in $\left.10^{6}(\mathrm{ppm})\right]$ and $A \simeq$ $1.1 \times 10^{-6}(1.1 \mathrm{ppm})$.

In conclusion, we have reported characterizations of low-loss mirrors by measurements of cavity-decay time, of cavity finesse, and of peak reflection. The lowest observed total loss (transmission plus absorption and scatter) was $1.6 \mathrm{ppm}$ per mirror at $852 \mathrm{~nm}$, which corresponds to a mirror reflectivity of 0.9999984 and a cavity $Q \simeq 1.8 \times 10^{10}$ for a $4-\mathrm{mm}$ cavity. Our measurements of finesse made by scanning the frequency of an incident laser yield $F \simeq$ $2 \times 10^{6}$. Observations of cavity reflection indicate that the mirror loss as a result of transmission is of comparable magnitude to the loss from absorption plus scatter. Mirrors such as these should have a wide impact in a variety of applications ranging from practical devices such as laser gyroscopes and high-resolution optical spectrum analyzers to fundamental and novel experiments in quantum optics, measurement science, and astrophysics.

This research was supported by the National Science Foundation (PHY-9014547), the Venture Research Unit of British Petroleum, and by the U.S, Office of Naval Research (N00014-90-J-1058). The technical assistance of E. Polzik is gratefully acknowledged.

\section{References}

1. K. Scribner, General Optics, Inc., 554 Flinn Avenue, Moorpark, California 93021 (personal communication, 1991).

2. J. R. Sites, P. Gilstrap, and R. Rujkorakarn, Opt. Eng. 22, 447 (1983).

3. R. Lalezari and R. G. Knollenberg, Proc. Soc. PhotoOpt. Instrum. Eng. 1164, 222 (1989).

4. D. Z. Anderson, J. C. Frisch, and C. S. Masser, Appl. Opt. 23, 1238 (1984), and references therein. 